\title{
Transcriptional responses of Daphnis nerii larval midgut to oral infection by Daphnis nerii cypovirus-23
}

\author{
Wendong Kuang ${ }^{1 \dagger}$, Chenghua Yan ${ }^{3 \dagger}$, Zhigao Zhan ${ }^{1 \dagger}$, Limei Guan ${ }^{1}$, Jinchang Wang ${ }^{1}$, Junhui Chen ${ }^{1}$, \\ Jianghuai Li ${ }^{1}$, Guangqiang $\mathrm{Ma}^{3^{*}}, \mathrm{Xi} \mathrm{Zhou}^{1,2^{*}}$ and Liang Jin ${ }^{1^{*}}$
}

\begin{abstract}
Background: Daphnis nerii cypovirus-23 (DnCPV-23) is a new type of cypovirus and has a lethal effect on the oleander hawk moth, Daphnis nerii which feeds on leave of Oleander and Catharanthus et al. After DnCPV-23 infection, the change of Daphnis nerii responses has not been reported.

Methods: To better understand the pathogenic mechanism of DnCPV-23 infection, 3rd-instar Daphnis nerii larvae were orally infected with DnCPV-23 occlusion bodies and the transcriptional responses of the Daphnis nerii midgut were analyzed $72 \mathrm{~h}$ post-infection using RNA-seq.

Results: The results showed that 1979 differentially expressed Daphnis nerii transcripts in the infected midgut had been identified. KEGG analysis showed that protein digestion and absorption, Toll and Imd signaling pathway were down-regulated. Based on the result, we speculated that food digestion and absorption in insect midgut might be impaired after virus infection. In addition, the down-regulation of the immune response may make $D$. nerii more susceptible to bacterial infections. Glycerophospholipid metabolism and xenobiotics metabolism were up-regulated. These two types of pathways may affect the viral replication and xenobiotic detoxification of insect, respectively.

Conclusion: These results may facilitate a better understanding of the changes in Daphnis nerii metabolism during cypovirus infection and serve as a basis for future research on the molecular mechanism of DnCPV-23 invasion.
\end{abstract}

Keywords: Daphnis nerii cypovirus-23, Midgut, Transcriptome analysis

\section{Introduction}

The oleander hawk moth, Daphnis nerii (D. nerii), belongs to Lepidoptera, Sphingidae family, is a worldwide pest [1]. D. nerii larvae damages leave of Oleander, Catharanthus, Vinca, Adenium, Vitis, Tabernaemontana,

\footnotetext{
*Correspondence: maguangqiang@163.com; zhouxi@wh.iov.cn; jinliang079@163.com

tWendong Kuang, Chenghua Yan and Zhigao Zhan have contributed equally to this work

${ }^{1}$ Institute of Microbiology, Jiangxi Academy of Sciences, No. 7777 Changdong Road, Nanchang 330096, China

${ }^{3}$ School of Life Sciences, Jiangxi University of Traditional Chinese Medicine, Nanchang 330004, China

Full list of author information is available at the end of the article
}

Gardenia, Trachelospermum, Amsonia, Asclepias, Carissa, Rhazya, Thevetia, Jasminum and Ipomoea [2, $3]$, which affect the landscape and the medicinal value of these plants. At present, the chemical pesticide decamethrin is used to control D. nerii [2].

Cypovirus is a member of the Reoviridae family, and is characterized by its single layered capsid [4]. DnCPV-23 was isolated from naturally diseased $D$. nerii larvae. This was a new type of cypovirus based on different electrophoretic migration patterns and conserved terminal sequences $[1,5,6]$. In addition to Daphnis nerii, it has been found that DnCPV-23 can also induce infection and death in many species of Sphingidae insects, such 
as Cephonodes hylas Linnaeus, Ampelophaga rubiginosa Bremer \& Grey, and Agathia lycaenaria Kollar. The genome of DnCPV-23 consists of ten segments of linear double-stranded RNA, referred to as genomic segments 1 (S1) to 10 (S10), in accordance with the fragments from longest to shortest [7]. Our previous research and unpublished data demonstrated that the virus could successfully replicate on the Sf9 [8] and Manduca sexta cell lines QB-MS 2-2 [9]. However, the molecular mechanism of the interactions between the new type cypovirus and its hosts remains unclear. It is necessary to identify the interactions between the virus and its hosts to achieve an in-depth understanding and reveal the exploitation potential of the virus for future insecticide development.

Recently, many studies in the field have generated large amounts of data using the aforementioned highthroughput approaches, from the silkworms or $\mathrm{BmN}$ cells infected with BmCPV, including (1) The possible host's RNAi response against BmCPV challenge in persistent and pathogenic Bombyx mori model was compared. During the pathogenic infection, it was found that higher level RNAi responses against BmCPV were observed, which further demonstrated the importance of RNAi as an antiviral mechanism [10]. (2) Gene expression profiles [11-19], DNA methylation [20], and lipidomic profile [21] of silkworm midgut or BmN cells after $\mathrm{BmCPV}$ infection were analyzed. These results suggested that many genes (for example, genes expressing Calreticulin, FK506-binding protein, and protein kinase $\mathrm{c}$ inhibitor gene, microRNAs, and activated protein kinase $C$ ) may play important roles in BmCPV replication. In addition, epigenetic regulation may influence silkworm-virus interaction, and BmCPV may modulate the lipid metabolism of cells for their self-interest.

Until now, the molecular mechanism underlying the midgut infection of DnCPV-23 is not clearly understood. Furthermore, since transcriptome analyses regarding $D$. nerii or DnCPV-23 have not yet been performed, this study aims to fill this gap about the new type cypovirus. The data and analysis presented here provide insights into the possible mechanism of DnCPV-23 infection and host defense and a basis for future DnCPV-23 relevant studies.

\section{Materials and methods}

\section{Daphnis nerii larval midgut and virus stock}

Newly wild-caught second instar larvae with a similar mass were used in this research investigation for the virus infection. Before infection, the $D$. nerii were supplied with 12 -h day/night cycles under $50 \pm 5 \%$ relative humidity conditions and were nurtured on oleander leaves at $27 \pm 1{ }^{\circ} \mathrm{C}$ for three days. The midgut tissues were collected from four pathogenically infected larvae at $72 \mathrm{~h}$
$[13,15]$ after feeding with DnCPV-23. The same tissues were also collected from three uninfected control larvae at the same time point. DnCPV was originally isolated from the larvae of $D$. nerii and propagated in $D$. nerii larvae [1]. The polyhedra suspension of DnCPV-23 utilized for infecting the $D$. nerii was stored at $4{ }^{\circ} \mathrm{C}$ in the dark.

\section{Virus inoculation}

In this study, the DnCPV-23 viral stock was suspended in distilled water at a concentration of $2 \times 10^{7}$ polyhe$\mathrm{dra} / \mathrm{mL}$. Then, $100 \mu \mathrm{L}$ of the viral suspension was spread evenly on one piece of oleander leaf measuring approximately $4 \mathrm{~cm} \times 1.5 \mathrm{~cm}$ each in size. The leaf was then fed to four $D$. nerii larvae. The dose of infection was calculated as $2 \times 10^{6}$ polyhedra per larva. In addition, three control larvae were fed the same quantity of leaves treated with only distilled water. After approximately $12 \mathrm{~h}$, fresh oleander leaves were used to feed the inoculated larvae after the DnCPV-23-inoculated leaves had been completely consumed.

\section{Sample preparation}

The midguts of both DnCPV-23-infected and control larvae were collected at $72 \mathrm{~h}$ post-inoculation by dissecting the larvae on ice. The isolated midgut was then quickly washed in $0.8 \%$ diethylpyrocarbonate (DEPC)-treated physiologic saline solution to remove the attached leaf pieces, and then frozen in liquid nitrogen [13, 22].

\section{RNA sequencing}

All of the RNA-seq procedures were conducted by the Oebiotech Company (Shanghai, China). The total RNA was extracted from the $D$. nerii midgut tissue using TRIzol reagent (Invitrogen, USA) according to the manufacture's protocols. The RNA integrity and concentrations were checked using an Agilent 2100 Bioanalyzer (Agilent Technologies, USA). In addition, seven RNA samples (including three uninfected samples and four infected samples) with RNA integrity were used to construct the libraries. The cDNA libraries were prepared using a TruSeq RNA Sample Preparation Kit (Illumina, USA) according to the manufacturer's protocols. Thereafter, the obtained cDNA libraries were sequenced on the Illumina HiSeq2500 platform, which generated paired-end raw reads of $125 \mathrm{bp}$.

\section{De novo assembly and functional annotation}

The raw data was pretreated by discarding reads with adaptors and low quality (quality scores $<30$ ). Then, the raw data was assembled using Trinity software with default parameters for de novo transcriptome assembly. Transcripts that were not shorter than 300 bp were used for subsequent analysis. To obtain the functional 
annotations of predicted protein-coding sequences, we searched against various databases, including the NCBI non-redundant (NR) protein, SwissProt, and euKaryotic Orthologous Groups (KOG) using Blastx with an E-value $<10^{-5}$. The top hit was utilized to assign gene names. Whereafter, the Gene Ontology (GO) annotations of the transcripts were then analyzed based on SwissProt annotations, and functional classifications were assigned by WeGO software. In addition, for the purpose of determining the biological pathways involved, the KEGG pathway was annotated based on the KEGG Orthology (KO) identifiers.

\section{Differential gene expression analysis}

RNA sequencing results from the two groups were mapped to the assembled transcriptome using bowtie2 [23] and express [24]. The FPKM (fragments per kb per million reads) method [25] was utilized to calculate the expression levels of the unigenes, which eliminated the influencing effects of the different gene lengths and sequencing levels. The differences in the unigene expressions between the two groups were calculated with DESeq [26] and any significant differences were determined with $P<0.05$ and an absolute value of $\log 2$ fold change $>1$.

\section{Real-time quantitative reverse transcription PCR (Real-Time qRT-PCR)}

This study utilized qRT-PCR to analyze the expression level of DnCPV-23 S1, S10 genes of transcriptome samples, and verify the DEGs recognized by the RNA-seq. The total RNA was isolated from the samples of the transcriptomic analysis using TRIzol reagent (Life Technologies) and was then treated with DNase I (Fermentas, Glen Burnie, MD, USA). We reversely transcribed $1 \mu \mathrm{g}$ of the total RNA per sample into complementary DNA (cDNA) using a PrimeScript RT Reagent Kit (Takara). Then, qRT-PCR was performed using Talent qPCR PreMix SYBR Green (Tiangen, China) on a QuantStudio ${ }^{\mathrm{TM}}$ 7 Flex Real-Time PCR System (Applied Biosystems ${ }^{\mathrm{TM}}$ ). One cycle was added for melting curve analysis for all the reactions to verify the product specificity. The expression level of each gene relative to that of the RPL13 gene was calculated using the $2^{-\triangle \triangle \mathrm{CT}}$ method [27]. All of the primers for the aforementioned target genes are listed in Table 1. Results are representative of two to three independent experiments.

\section{Results}

\section{Virus infection of the samples}

Prior to the transcriptome analysis, qRT-PCR was used to detect the mRNA levels of the DnCPV-23 S1 and S10 genes in the infected and uninfected samples. The results showed that the infected group had been successfully infected based on the high relative expression of the viral gene mRNA compared with uninfected group (Fig. 1).

\section{Transcriptome sequencing and assembly}

The RNA-Seq data from the DnCPV-23-infected and control groups contained 346.39 million reads, and 334.60 million clean reads after trimming, among which 96.17 to $97.39 \%$ per sample were determined to be useful. The acquired clean reads were assembled into 31,696 unigenes (>300 bp). The average length of these unigenes was $1347.61 \mathrm{bp}$, and the N50 length was $2348 \mathrm{bp}$; other information about these unigenes were shown in Table 2. This study then assembled 31.696 unigenes ranging from $301 \mathrm{bp}$ to $32,420 \mathrm{bp}$. The total unigene length was $42,713,980$.

\section{Transcriptome annotation}

A total of 31,696 assembled unigenes were searched against the public databases, including the NR, Swissprot, KOG, GO, and KEGG databases, among which 16,820 (53.1\%) (Fig. 2) unigenes were annotated. The distribution patterns of the unigenes in the different databases were as follows: 16,615 unigenes in the NR database, 11,152 unigenes in the Swissprot database, 10,374 unigenes in the KOG, 10,468 unigenes in the GO, and 5501 unigenes in the KEGG databases (Table 3). Figure 2 shows the degree of overlap between the unigenes annotated in the different databases. It was found that 4353 (13.7\%) unigenes overlapped in all five databases, while $12,390(73.7 \%)$ unigenes overlapped in two or more databases.

\section{Significant impacts of the viral infection on the hosts' transcriptome expressions}

As shown in Fig. 3, the main component PCA1 had reached $41.56 \%$, and the main component PCA2 had reached $27.23 \%$. Therefore, the percentage total of the two was $68.79 \%$, which accounted for a high proportion and represented the overall population to a large extent. This study's principal component analysis manifested a clear separation of the samples with the two treatments (Fig. 3A), which indicated that the samples had good repeatability. The heat map of the gene expressions is presented in Fig. 3B. The results suggested that these DEGs could distinguish the samples. The results revealed that the viral infection could exert apparent influences on the midgut gene expressions. In addition, the transcriptome results showed that 1166 genes were down-regulated (accounting for $3.68 \%$ of the total assembled unigenes) and 812 genes (accounting for $2.56 \%$ of the total assembled unigenes) were up-regulated as a response to the DnCPV-23 infection (Fig. 3C). 
Table 1 Primers used in the qRT-PCR for the the viral RNA detection of transcriptome samples and validation of the RNA-seq

\begin{tabular}{|c|c|c|c|c|c|}
\hline No & Primer name & Primer sequence ( $5^{\prime}$ to $\left.3^{\prime}\right)$ & $\operatorname{Tm}\left({ }^{\circ} \mathrm{C}\right)$ & Gene id & Target gene \\
\hline & S1-RTPCR-F & GTGCTGATGGTCTGCTAA & 49.6 & N/A & DnCPV S1 \\
\hline & S1-RTPCR-R & TGATTGATGACGACATTGAG & 51.5 & & \\
\hline & S10-RTPCR-F & GTCCGCCAATACTCTCAG & 52.6 & N/A & DnCPV S10 \\
\hline & S10-RTPCR-R & CGTAGTCCATCGTCAATCA & 51.3 & & \\
\hline 1 & CASP8-F & ACTGGAGAAGACTATGAGGTTA & 51.5 & TRINITY_DN10280_c0_g1_i1_3 & CASP8 \\
\hline 2 & CASP8-R & ACGCTGTCATCTTGGCTAA & 53.7 & & \\
\hline 3 & CYP6AB13-F & GATTCACACCAGCATTCAG & 51.0 & TRINITY_DN11437_c0_g1_i1_6 & CYP6AB13 \\
\hline 4 & CYP6AB13-R & CAGTCGTATATCTCGCCATA & 50.5 & & \\
\hline 5 & CYP6B45-F & GCGATACCGAACCAGAAC & 53.4 & TRINITY_DN12532_C0_g7_i1_1 & CYP6B45 \\
\hline 6 & CYP6B45-R & ATTGGCAGTAAGTGTGAGTT & 51.0 & & \\
\hline 7 & DHRS4-F & TCTTCTATCGCCGCATATCA & 52.8 & TRINITY_DN12896_c1_g2_i3_3 & DHRS4 \\
\hline 8 & DHRS4-R & CACCACCTCATTAGCAATCG & 53.5 & & \\
\hline 9 & PNLIP-F & CACCTCGTAGACTTGGAAGA & 53.5 & TRINITY_DN12381_C0_g2_i1_6 & PNLIP \\
\hline 10 & PNLIP-R & GTTAGCGTTGCCATTGACA & 53.2 & & \\
\hline 11 & PRSS1_2_3-F & CCTGGAAGATGGCGTGTT & 55.4 & TRINITY_DN10836_C0_g5_i1_6 & PRSS1_2_3 \\
\hline 12 & PRSS1_2_3-R & TCGGCGGTAATTCGGTTAT & 53.5 & & \\
\hline 13 & $\mathrm{RDH} 12-\mathrm{F}$ & GTCTAATCGTCCGCTATTGAG & 52.5 & TRINITY_DN14445_C0_g1_i1_3 & $\mathrm{RDH} 12$ \\
\hline 14 & $\mathrm{RDH} 12-\mathrm{R}$ & CTGTAGGTGAAGATTGCCATT & 52.2 & & \\
\hline 15 & SCARB1-F & AACACAACAAGAGGCATCAC & 53.0 & TRINITY_DN14140_c0_g1_i1_6 & SCARB1 \\
\hline 16 & SCARB1-R & GTCGTCGGTTCAATATCCATAA & 51.7 & & \\
\hline 17 & SLC46A1-F & TGGAACGACACGACAAGT & 53.7 & TRINITY_DN8071_C0_g1_i2_5 & SLC46A1 \\
\hline 18 & SLC46A1-R & CAACAGAGTGCGAACAGTATA & 51.7 & & \\
\hline 19 & SLC52A3-F & AAGCGATTGTGGAAGATGTC & 52.5 & TRINITY_DN11521_C0_g1_i2_4 & SLC52A3 \\
\hline 20 & SLC52A3-R & CGGCATACACGAGTACGA & 54.4 & & \\
\hline 21 & $A B C A 3-F$ & CGATATACGCCGCAAGTAAG & 53.3 & TRINITY_DN12365_C0_g1_i6_2 & $A B C A 3$ \\
\hline 22 & $A B C A 3-R$ & GCAGTTCTCTACATTCAGTTGA & 51.8 & & \\
\hline 23 & ABCC4-F & AGTGGATGGAAGGTTGGAAT & 53.3 & TRINITY_DN11997_C1_g1_i24_2 & $A B C C 4$ \\
\hline 24 & $A B C C 4-R$ & CGGCTCTTGTGGTATAATTGA & 51.9 & & \\
\hline 25 & CYP6B6-F & GGACTATTGTTGGCGAATC & 50.7 & TRINITY_DN13898_C0_g1_i1_4 & CYP6B6 \\
\hline \multirow[t]{3}{*}{26} & CYP6B6-R & TTGTGGAAGAAGACGATGT & 50.5 & & \\
\hline & GAPDH-F & TATGTTCGTTGTCGGAGTTA & 50.1 & TRINITY_DN5984_C0_g1_i2_2 & GAPDH \\
\hline & GAPDH-R & TAGCAGTAGTGGCGTGTA & 52.4 & & \\
\hline 27 & LYPLA3-F & ACATCCACGACACAAGACTA & 52.8 & TRINITY_DN10250_C0_g1_i1_1 & LYPLA3 \\
\hline 28 & LYPLA3-R & GACCGATAATGAACTCCTGAAT & 51.5 & & \\
\hline 29 & NTE-F & CAGCCTGGAAGGTAAGTAGT & 53.6 & TRINITY_DN14343_CO_g2_i1_4 & NTE \\
\hline 30 & NTE-R & CTCATAGACGAGCGACAGT & 53.8 & & \\
\hline 31 & UGT-F & GCATTCATTCAAGTCCATCAG & 51.3 & TRINITY_DN14215_C0_g5_i7_5 & UGT \\
\hline 32 & UGT-R & GCCTCCATCAATAATCACCAA & 52.2 & & \\
\hline 33 & DnRPL13-F & GAACTATTGGCATTGCTGTTG & 52 & TRINITY_DN4717_CO_g1_i2_3 & RPL13 \\
\hline 34 & DnRPL13-R & TCCTCCTCATTGGCTTCAC & 54.5 & & \\
\hline
\end{tabular}

\section{Analysis of the differently expressed genes}

In this study, KEGG function enrichment analysis was performed on the differential genes expressed in the DnCPV-23-infected and uninfected control groups to clarify the relevant biological pathways involved in the differential genes. Among all of the DEGs, 298 DEGs had KEGG annotations, of which 118 were up-regulated genes and 180 were down-regulated genes. According to the pValue of KEGG analysis of up-regulated and down-regulated signal pathways, we identified 20 most significant signal pathways each. These pathways play an important role in insect reproduction, immunity, digestion and absorption and xenobiotic metabolism and so on (Fig. 4). 
A

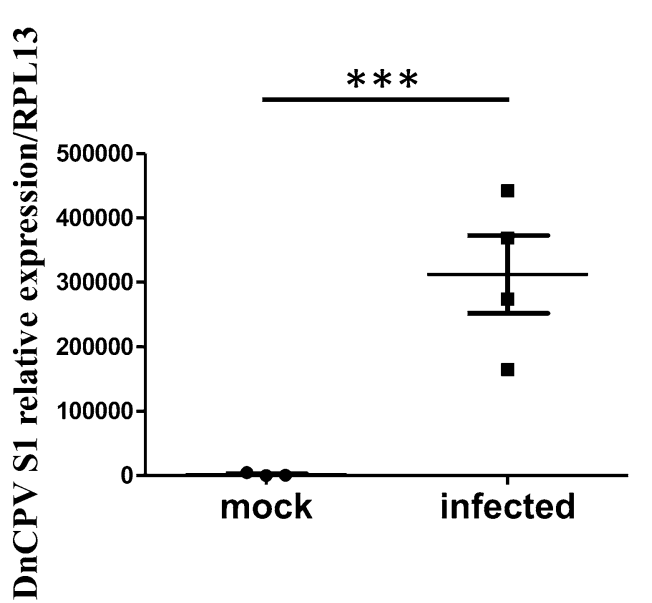

$\mathrm{B}$

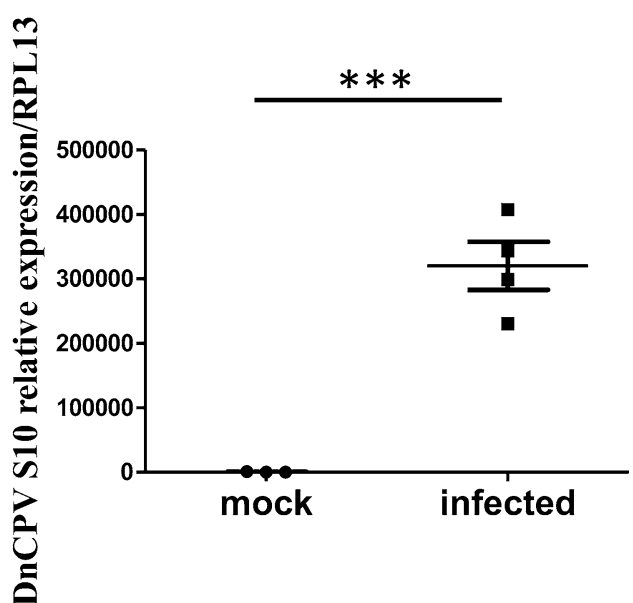

Fig. 1 Detection of the viral RNA in transcriptome samples at 72 hpi (hours post infection). After feeding for $72 \mathrm{~h}$, the mRNA levels of DnCPV-23 S1 $(\mathbf{A})$ and $S 10(\mathbf{B})$ in the midgut of $D$. nerii were detected. The asterisk $\left(^{* * *}\right)$ denotes the presence of a statistically significant difference $(p<0.001)$ by unpaired Student's t test

Table 2 Statistics of the assembly results

\begin{tabular}{lllllllll}
\hline Term & All & $\mathbf{>}=\mathbf{5 0 0} \mathbf{b p}$ & $\mathbf{>}=\mathbf{1 0 0 0} \mathbf{b p}$ & $\mathbf{N 5 0}$ & Total_Length & Max_Length & Min_Length & Average_Length \\
\hline Unigene & 31,696 & 20,703 & 12,663 & 2348 & $42,713,980$ & 32,420 & 301 & 1347.61 \\
\hline
\end{tabular}

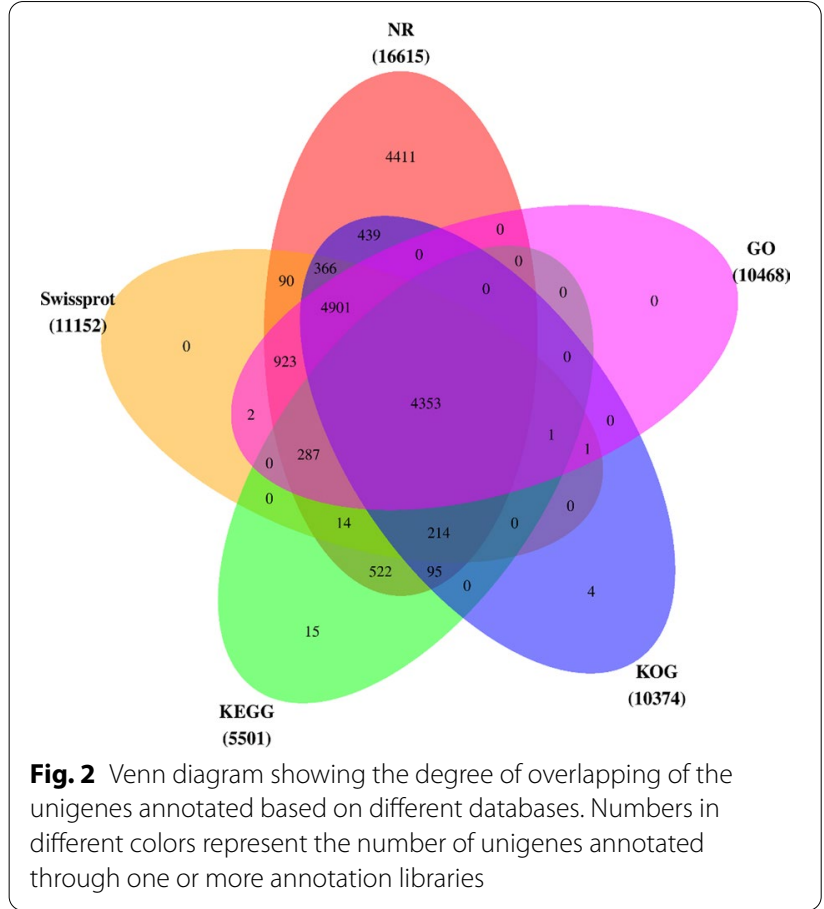

\section{qRT-PCR validation of DEGs}

To verify the reliability of the transcriptome data and the DEG results obtained by RNA-seq, seventeen DEGs were selected for qPCR analysis. As shown in Fig. 5, the fold-change values of DnCPV_1 sample vs Mock_1 sample obtained in the qPCR analysis results were consistent with the values obtained by the RNA-seq for all of the selected genes.

\section{Discussion}

This study analyzed the transcriptome of the uninfected $D$. nerii midgut and the DnCPV-23-infected $D$. nerii midgut presented unique gene expression profiles induced by DnCPV-23 infection for the first time. In addition, KEGG function enrichment analysis was performed on the differential genes expressed after DnCPV-23 infection. Compared with uninfected D. nerii midgut, the transcriptome profiles of the infected samples displayed universally changed transcript abundances for many pathways. 
Table 3 Annotation statistics for each database

\begin{tabular}{llll}
\hline Anno_Database & Annotated_Number & $\mathbf{3 0 0}<=$ length $<\mathbf{1 0 0 0}$ & Length $>=1 \mathbf{0 0 0}$ \\
\hline NR & $16,615(52.42 \%)$ & $6217(19.61 \%)$ & $10,398(32.81 \%)$ \\
Swissprot & $11,152(35.18 \%)$ & $2921(9.22 \%)$ & $8231(25.97 \%)$ \\
KEGG & $5501(17.36 \%)$ & $1694(5.34 \%)$ & $3807(12.01 \%)$ \\
KOG & $10,374(32.73 \%)$ & $2758(8.70 \%)$ & $7616(24.03 \%)$ \\
eggNOG & $15,249(48.11 \%)$ & $5239(16.53 \%)$ & $10,010(31.58 \%)$ \\
GO & $10,468(33.03 \%)$ & $2670(8.42 \%)$ & $7798(24.60 \%)$ \\
Pfam & $10,594(33.42 \%)$ & $2505(7.90 \%)$ & $8089(25.52 \%)$ \\
\hline
\end{tabular}

A

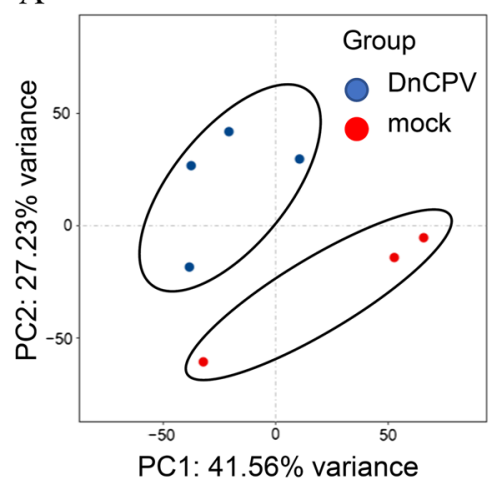

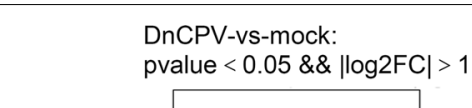

B

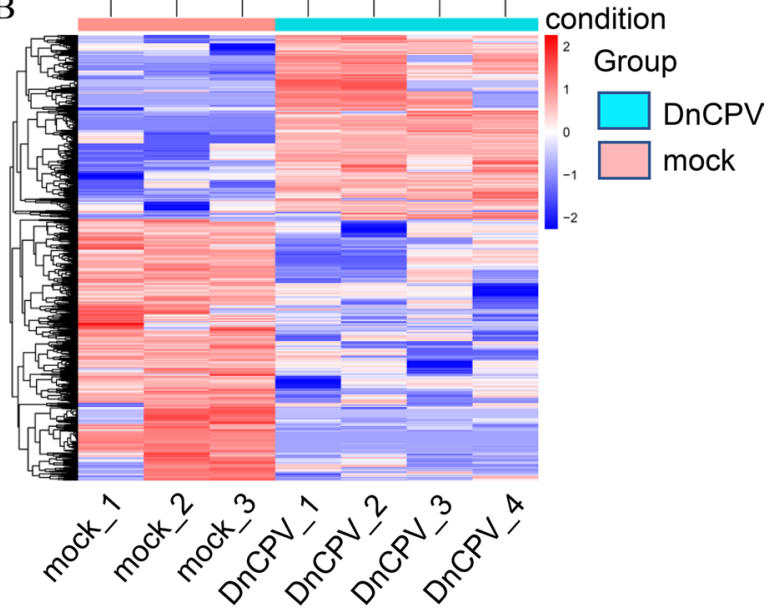

$\mathrm{C}$

Statistic of Differently Expressed mRNA

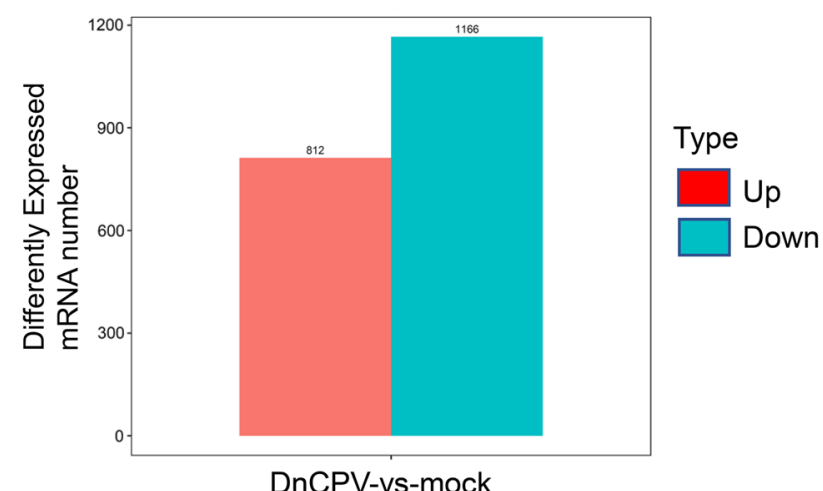

Fig. 3 Influence of DnCPV-23 infection on D. nerii transcriptome: A Plot of the 1st and 2nd principal component of the sample variations using the principal component analysis, in which the red dots represent samples without DnCPV-23 infection, and the green dots denote infected samples. B Heat map of 1,978 differently expressed genes (DEGs) in the infected samples and controls. C After infection, 812 genes were up-regulated (red bars) and 1166 genes were down-regulated (blue bars) 


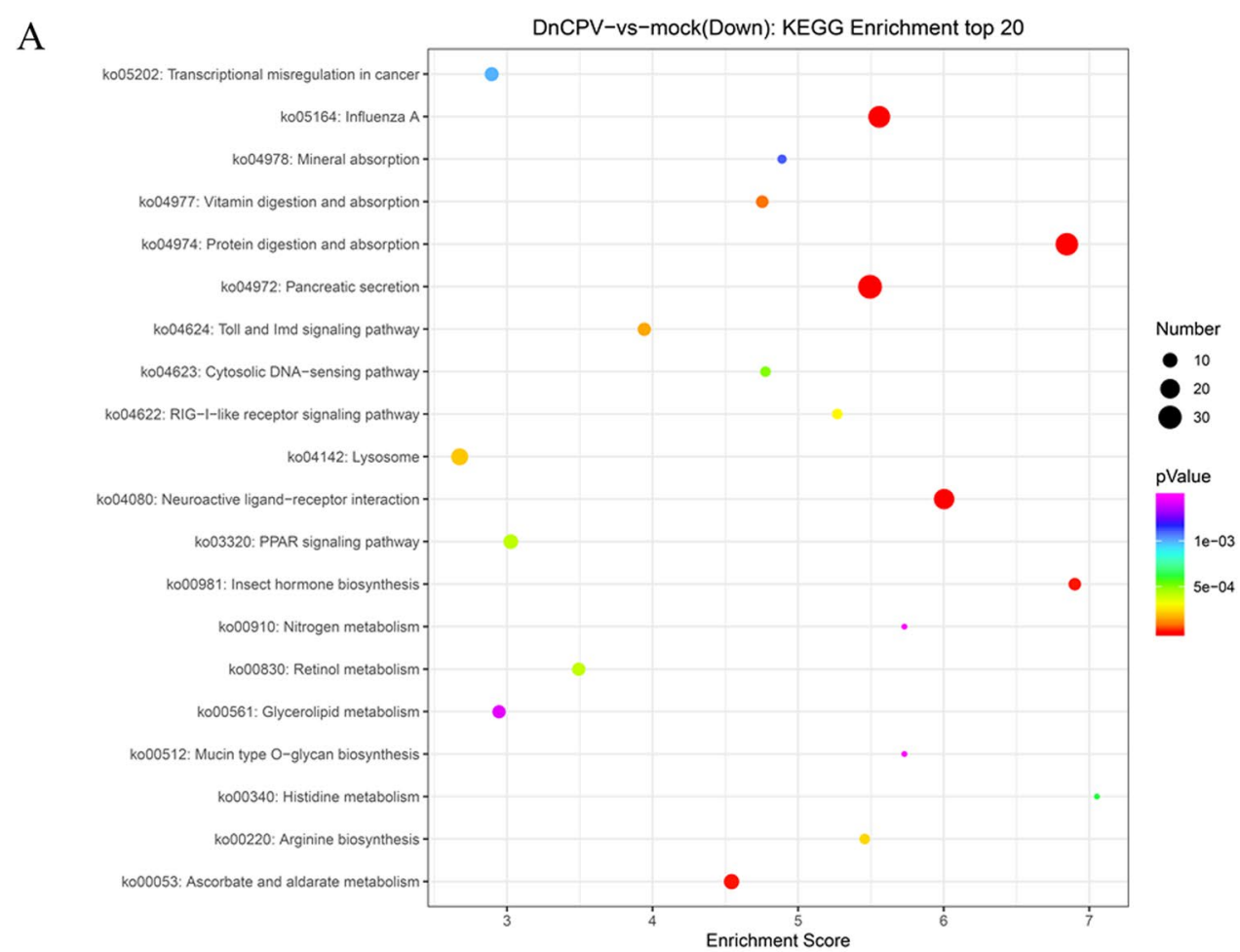

$\mathrm{B}$

DnCPV-vs-mock(Up): KEGG Enrichment top 20

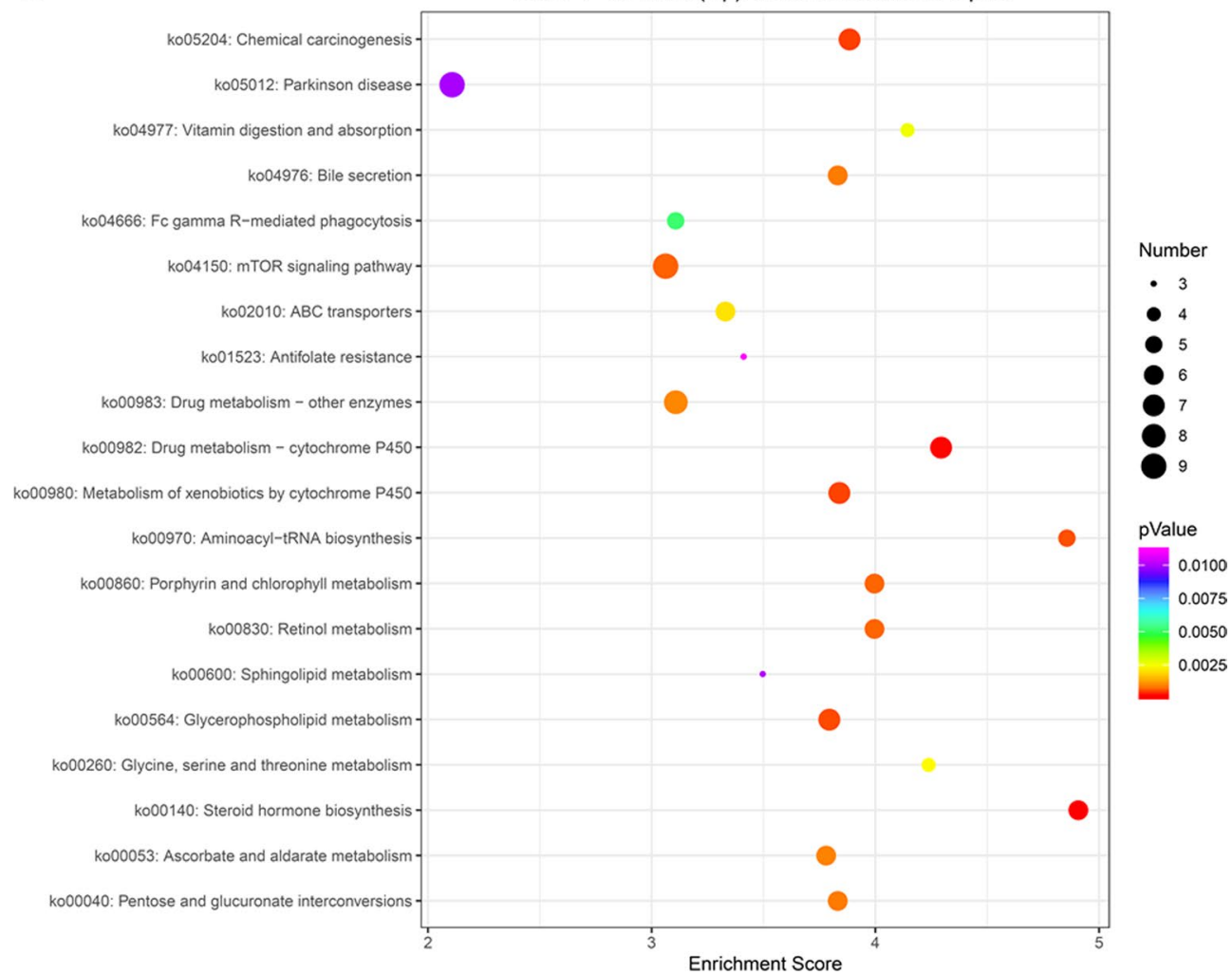

Fig. 4 KEGG classifications of DEGs after DnCPV-23 infection (Top 20): A. Down-regulated pathways; B. Up-regulated pathways. Horizontal axis of the figure is the enrichment score. The larger the bubble, the more the number of DEGs. The bubble color changes from purpl E-blu E-green-red, indicating that the smaller the enrichment pValue and the greater the significance 


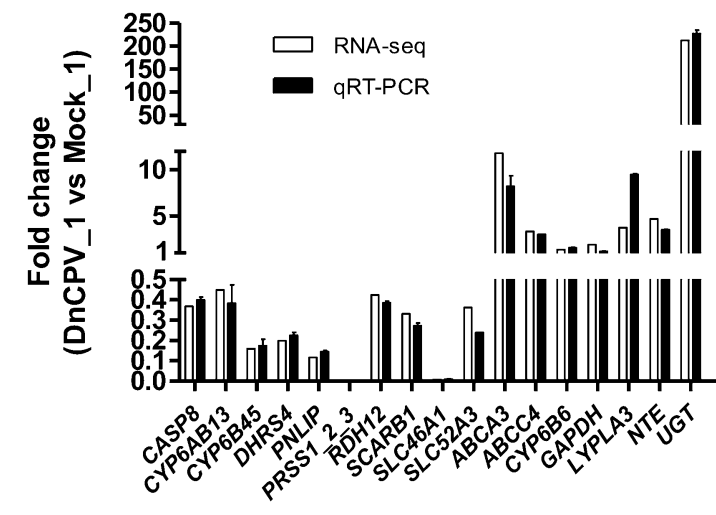

Fig. 5 Validation of RNA-seq profiles by real-time qPCR. To validate the RNA-seq data, the relative mRNA levels of 17 selected DEGs in the DnCPV_1 sample were examined by qPCR; The mRNA levels by qPCR are presented as the fold change compared with the Mock_1 sample after normalization against $R P L 13$. The relative expression levels from the RNA-seq analysis were calculated as RPKM values. Error bars show mean \pm SEM

Based on the pValue of KEGG analysis regarding up-regulated and down-regulated signal pathways, we identified 20 most significant signal pathways each. Among these signal pathways, the retinol metabolism pathway, vitamin digestion, and absorption signal pathway were down-regulated, consistent with the transcriptome study about BmCPV infected midgut vs non-infected midgut [13]. In addition, protein digestion and absorption pathway way was down-regulated in accord with previous research [10]. DnCPV infection may destroy the functions of digestion and the absorption of midguts, which causes the disturbance of protein and amino acid metabolism in D. nerii [13, 28]. Peptidoglycan recognition proteins (PGRPs) are pattern recognition molecules that are conserved from insects to mammals. PGRPs are the first receptors known to recognize, bind, or catalytically cleave the pathogenic microorganisms [29], PGRPs recognize bacteria and their unique cell wall component, eptidoglycan [30, 31]. This study observed nine transcripts of $D$. nerii isoforms of PGRP genes. Six transcripts were found to be down-regulated in the infected $D$. nerii midgut. The most highly expressed and most dramatically downregulated was TRINITY_DN13195_c0_g1_i3_3, which was down-regulated by as much as 51-fold. The downregulation of $P G R P$ expression can lead to a decrease in the ability of the $D$. nerii's innate immune system to recognize bacterial peptidoglycans (PGN), which may lead to $D$. nerii more susceptible to bacterial infections. In addition, BmPGRP-S2 was up-regulated upon $\mathrm{BmCPV}$ infection, overexpression of which can activate the Imd pathway and induce increased AMPs to enhance the antiviral capacity of transgenic silkworm against BmCPV [32]. Moreover, previous study demonstrates [33] that PGRPS2-1 and PGRPS2-2 can prevent $B m C P V$ replication. Based on this work, was speculated that the down-regulation of $P G R P$ was conducive to the replication of DnCPV-23.The gene CASP8 (KEGG gene name: caspase-8, Gene id: TRINITY_DN10280_c0_g1_ i1_3) (Dredd in Drosophila) was down-regulated more than two folds, and other caspase genes changed nonsignificantly. It is predicted to be involved in the cleavage of Relish, the Drosophila homolog of mammalian $\mathrm{NF}-\kappa \mathrm{B}$, resulting in activating the immune-deficient pathway (IMD)-induced expression of antimicrobial peptides in response to Gram-negative bacteria [3436], fungi and viruses [37]. Research performed by $\mathrm{Li}$ et al. proved BmDredd interacts with BmSTING to enhance antiviral signaling [38]. The down-regulation of this gene may be very important for DnCPV-23 to escape from the host innate immune system and replicate in the midgut. Our result conflicted with the work by Guo et al. [11]. We speculated the contradiction might be related to the different stages of virus-host interaction or the heterogeneity of different species against virues. The pathways and the genes mentioned above are listed in Table 4 (The expression of genes in each sample is shown in Additional file 1).

In this study, the up-regulation of glycerophospholipid metabolism was consistent with Zhang's research [21]. The up-regulation of this pathway may be related to the viral replication $[39,40]$. In addition, Glycine, serine and threonine metabolism were up-regulated in this transcriptome analysis. In the study by $\mathrm{Wu}$ et al., two genes related to this signaling pathway were upregulated and the other down-regulated. In our study, the expression levels of the phosphoserine phosphatase genes were significantly higher in DnCPV-23-infected midgut than in the non-infected group, suggesting that serine metabolism disorders were induced after DnCPV-23 infection. Expression of many UGT genes was up-regulated; UDP-glucuronosyltransferase (UGT) isozymes take endogenic and exogenic toxic substances as substrates, catalyze detoxification of many chemical toxins in our daily diet and environment by conjugation to glucuronic acid or glucose [41, 42]. After DnCPV-23 infection, it was speculated that the $D$. nerii tended to strengthen the elimination of lipophilic endobiotics such as hormones and xenobiotics including phytoalexins and drugs conjugated by invertebrates and plants mainly with glucose [42] through promoting the transcription of $U G T s$ by regulating the activities of nuclear-receptor family (CAR, PXR, FXR, LXR, and PPAR), the arylhydrocarbon receptor [43] or ubiquitous transcription factors (FOXA1, Sp1, and Cdx2) 


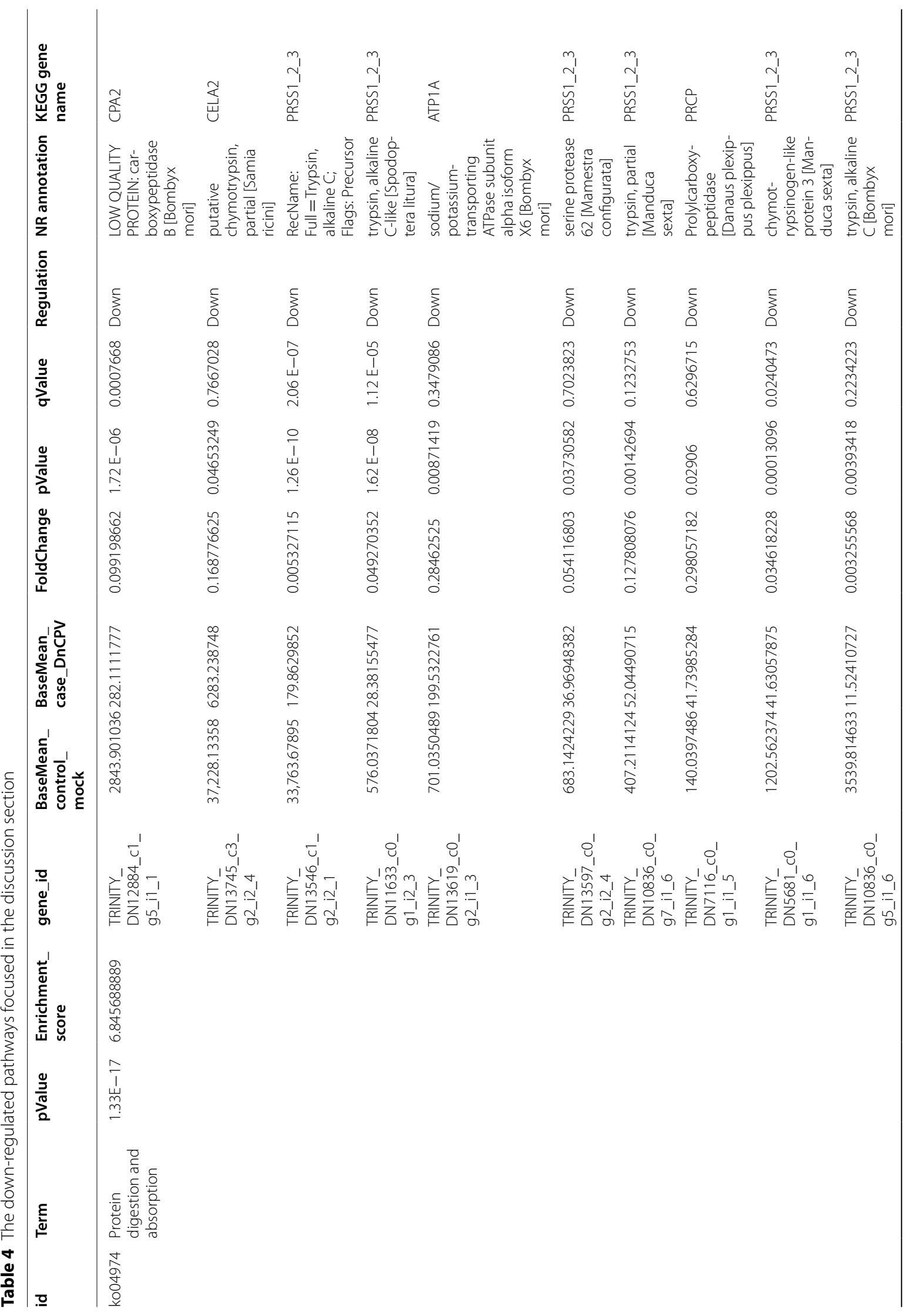




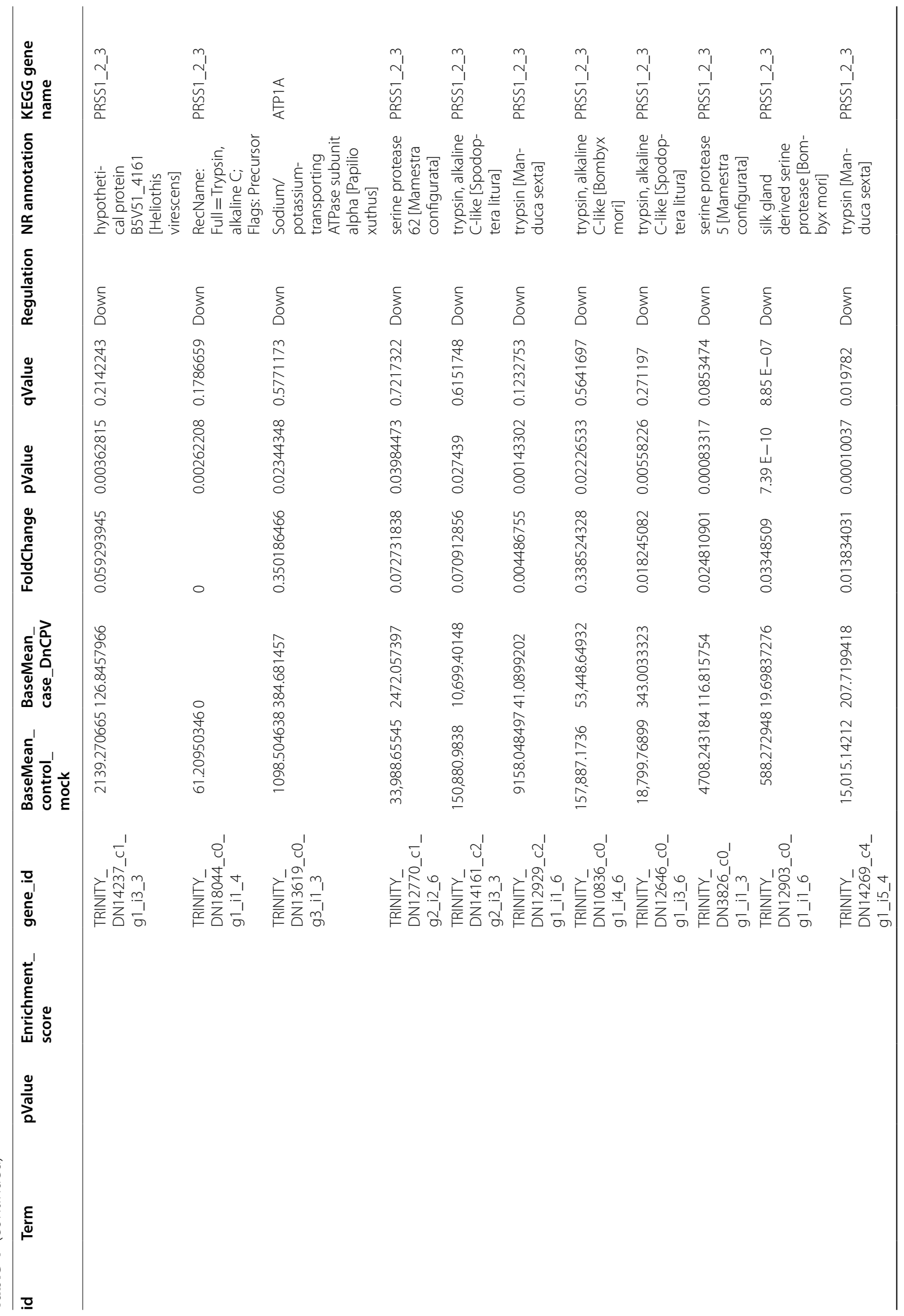




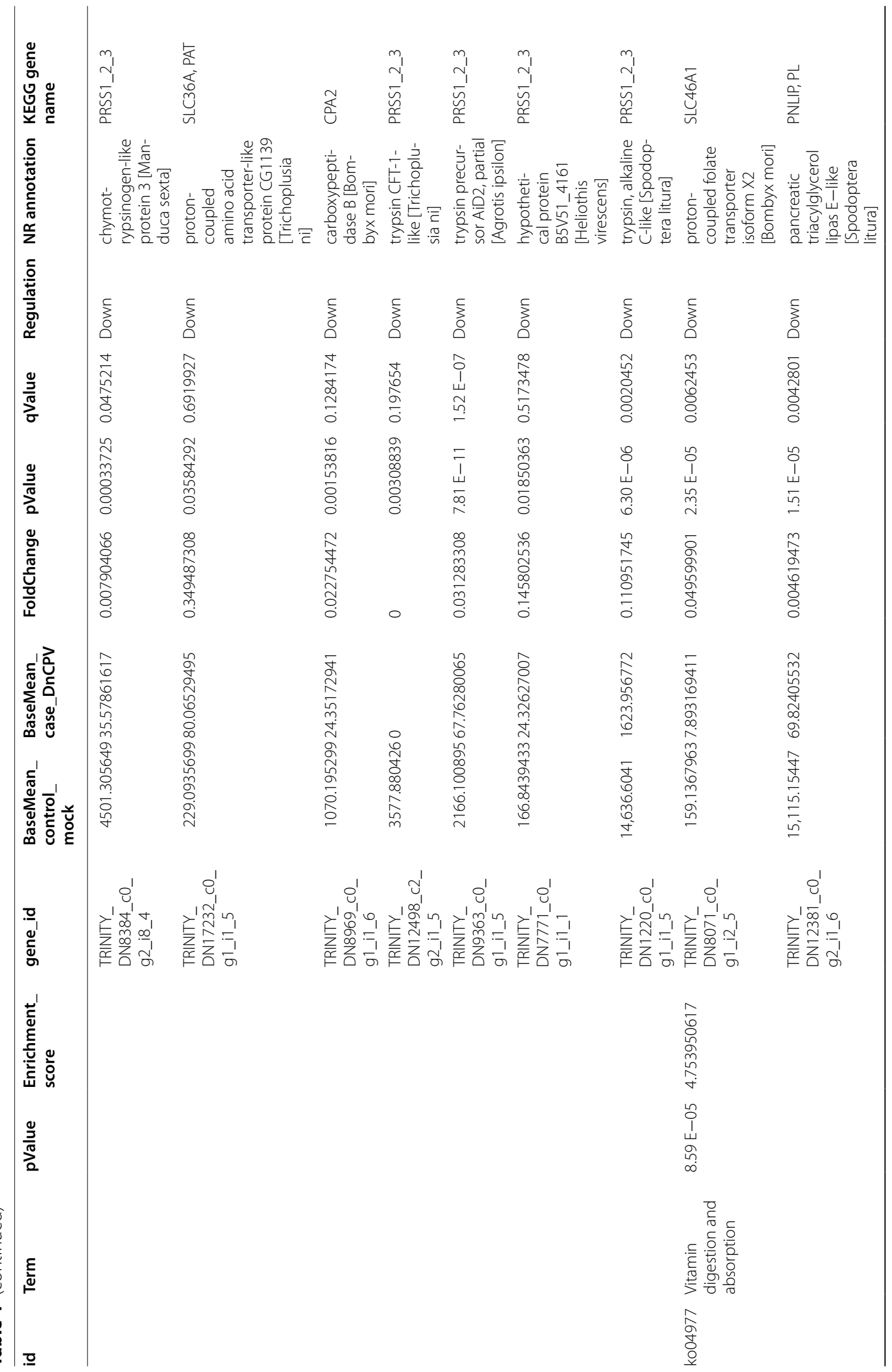




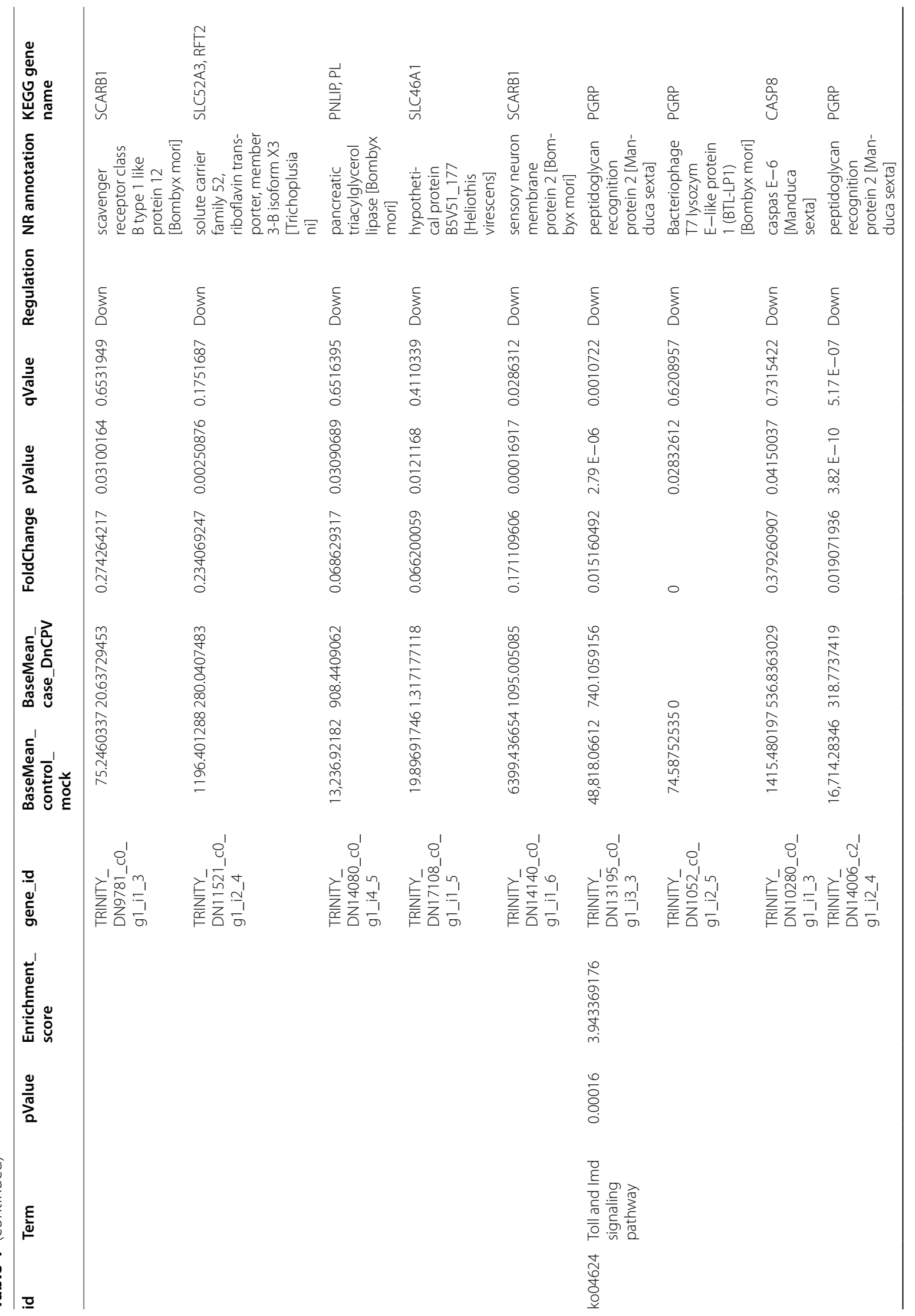




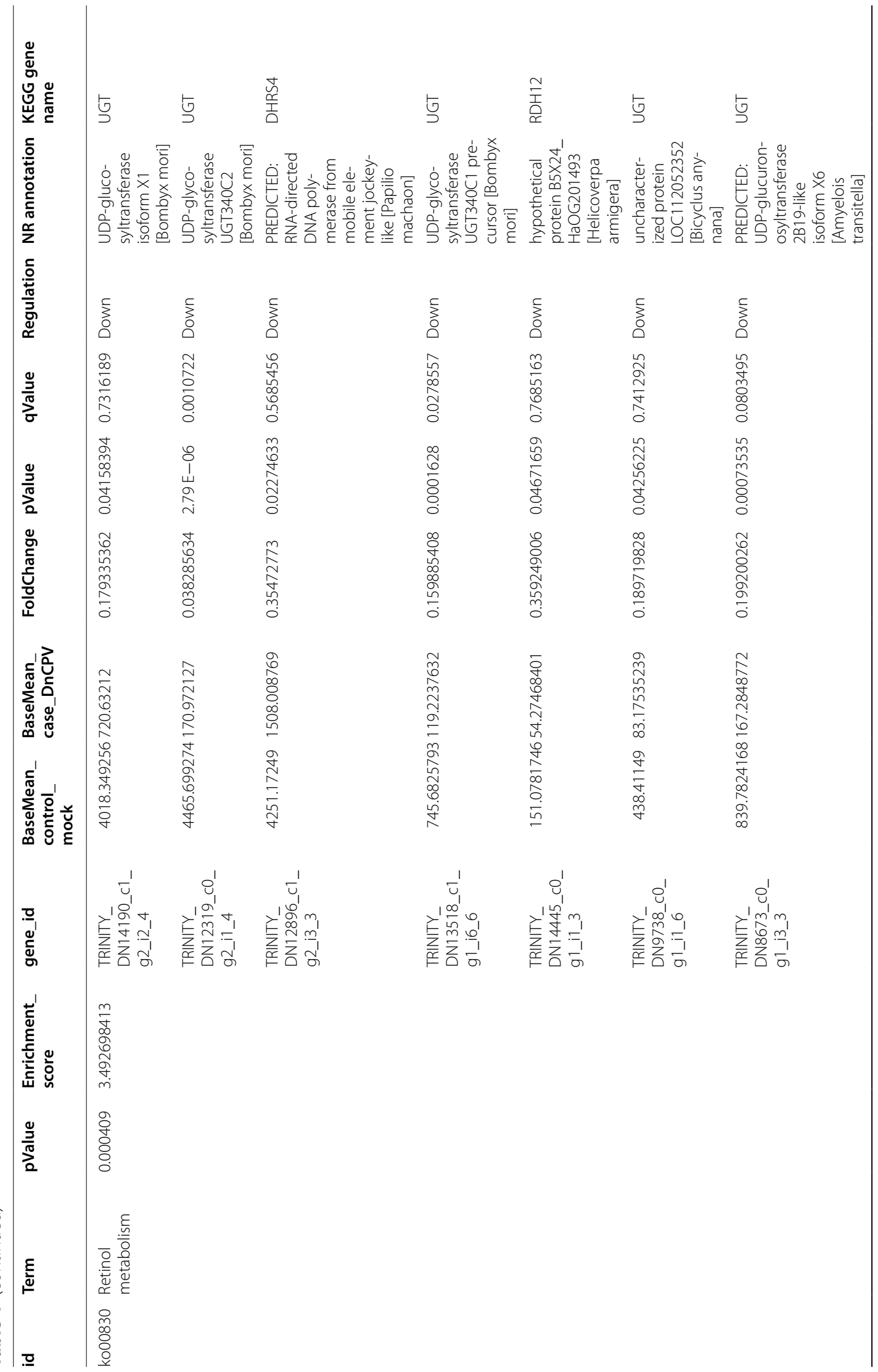




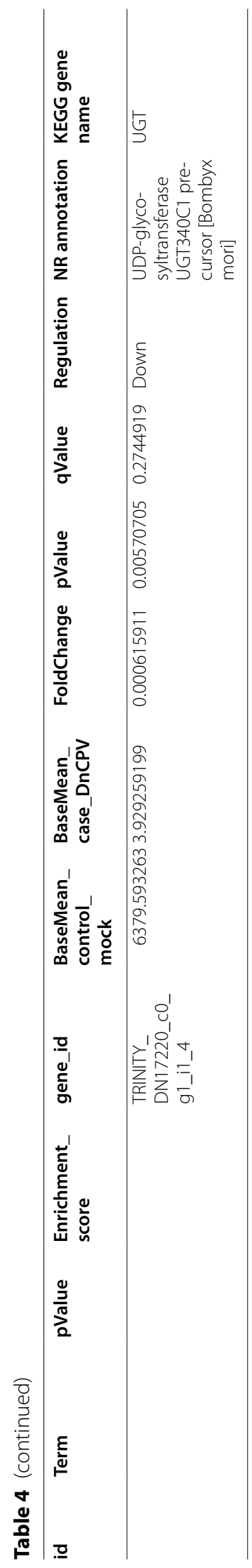




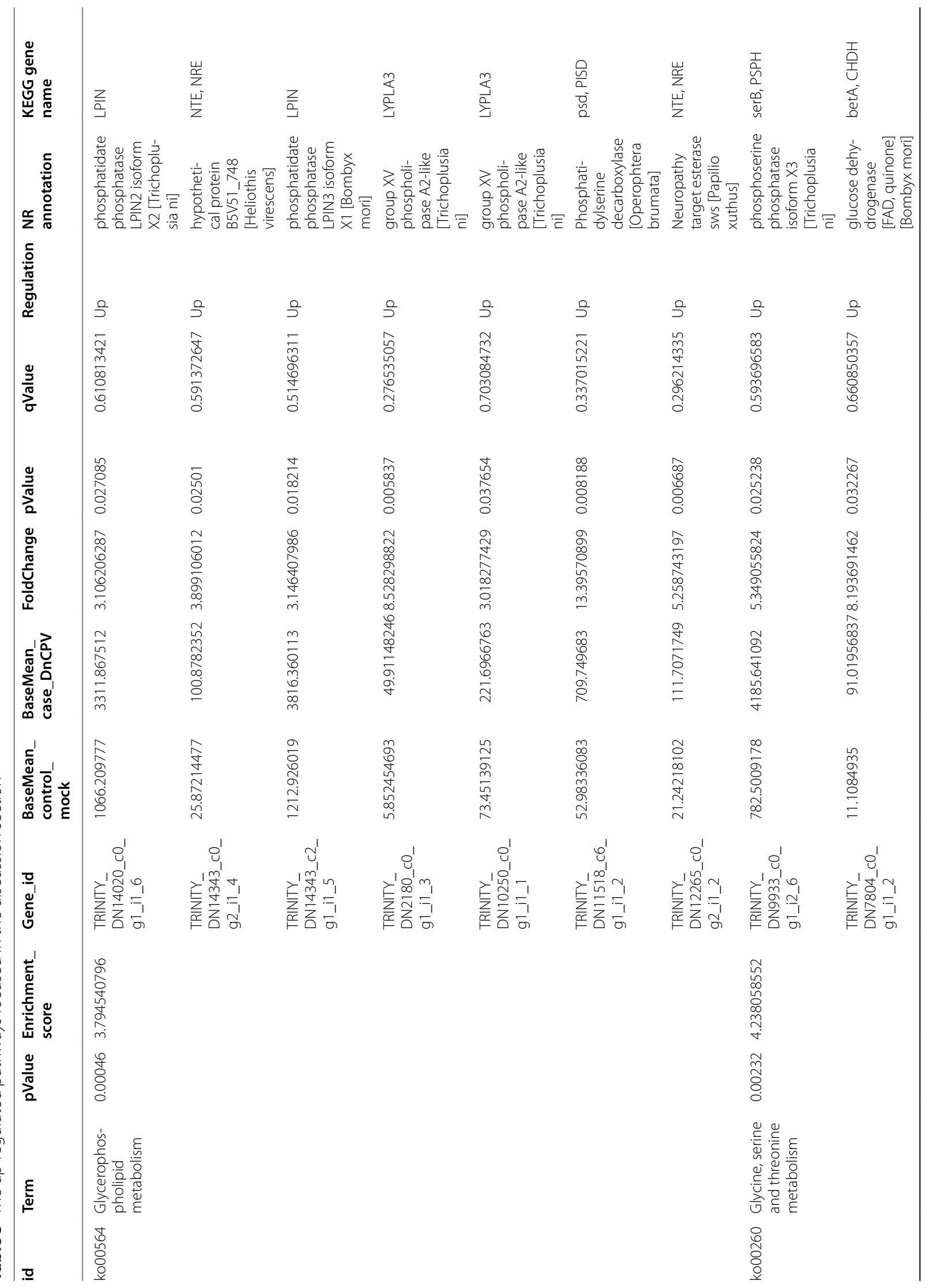




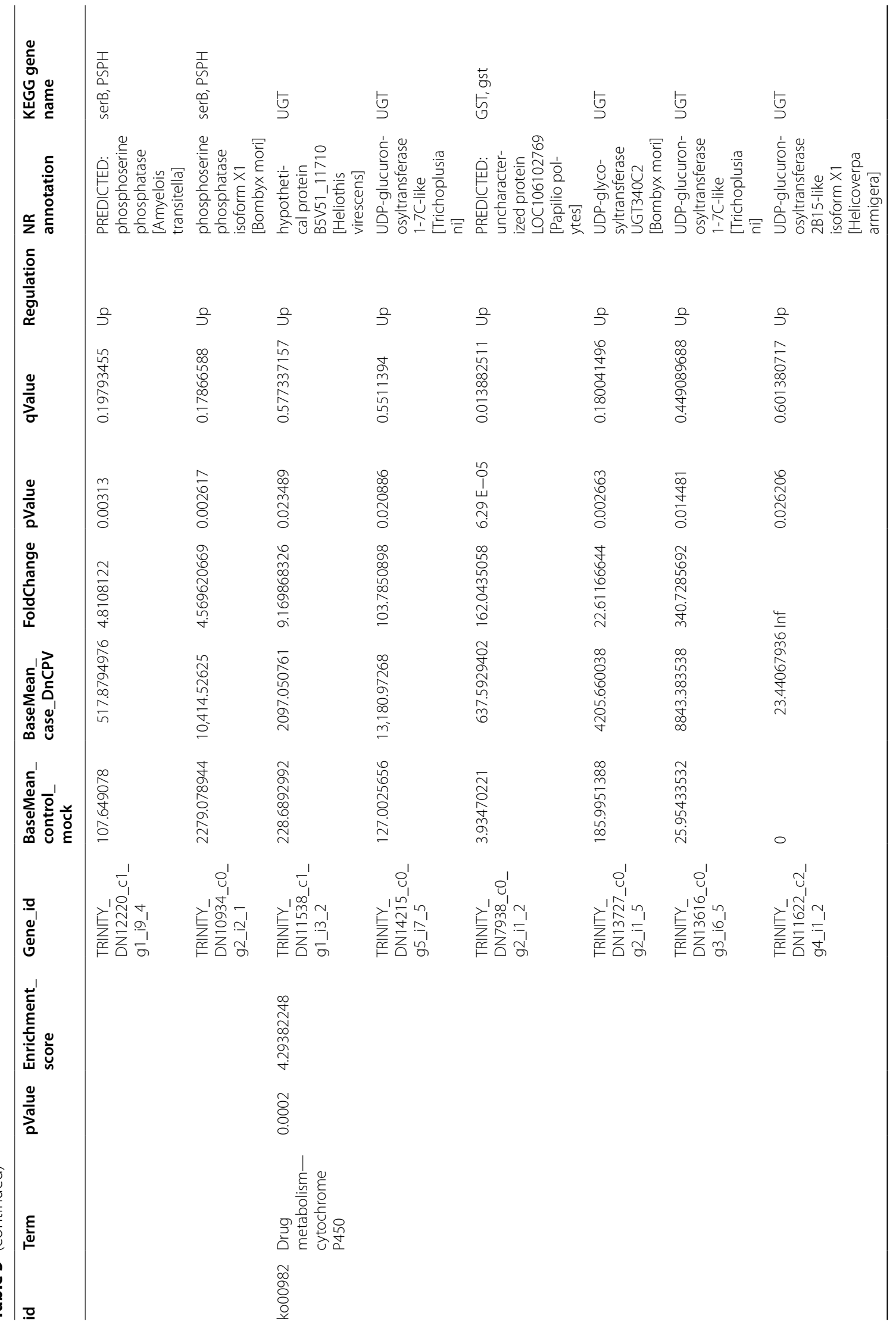




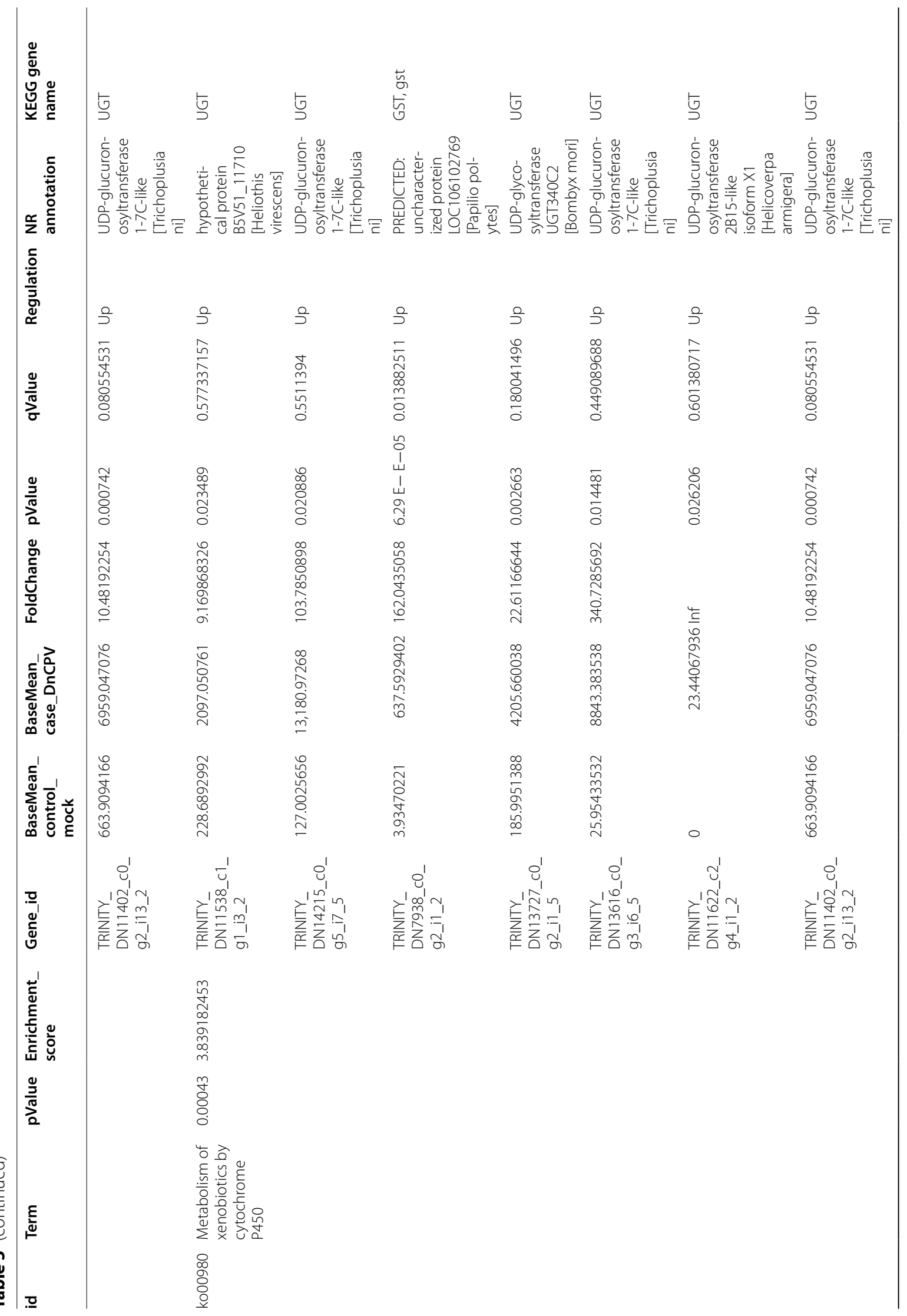




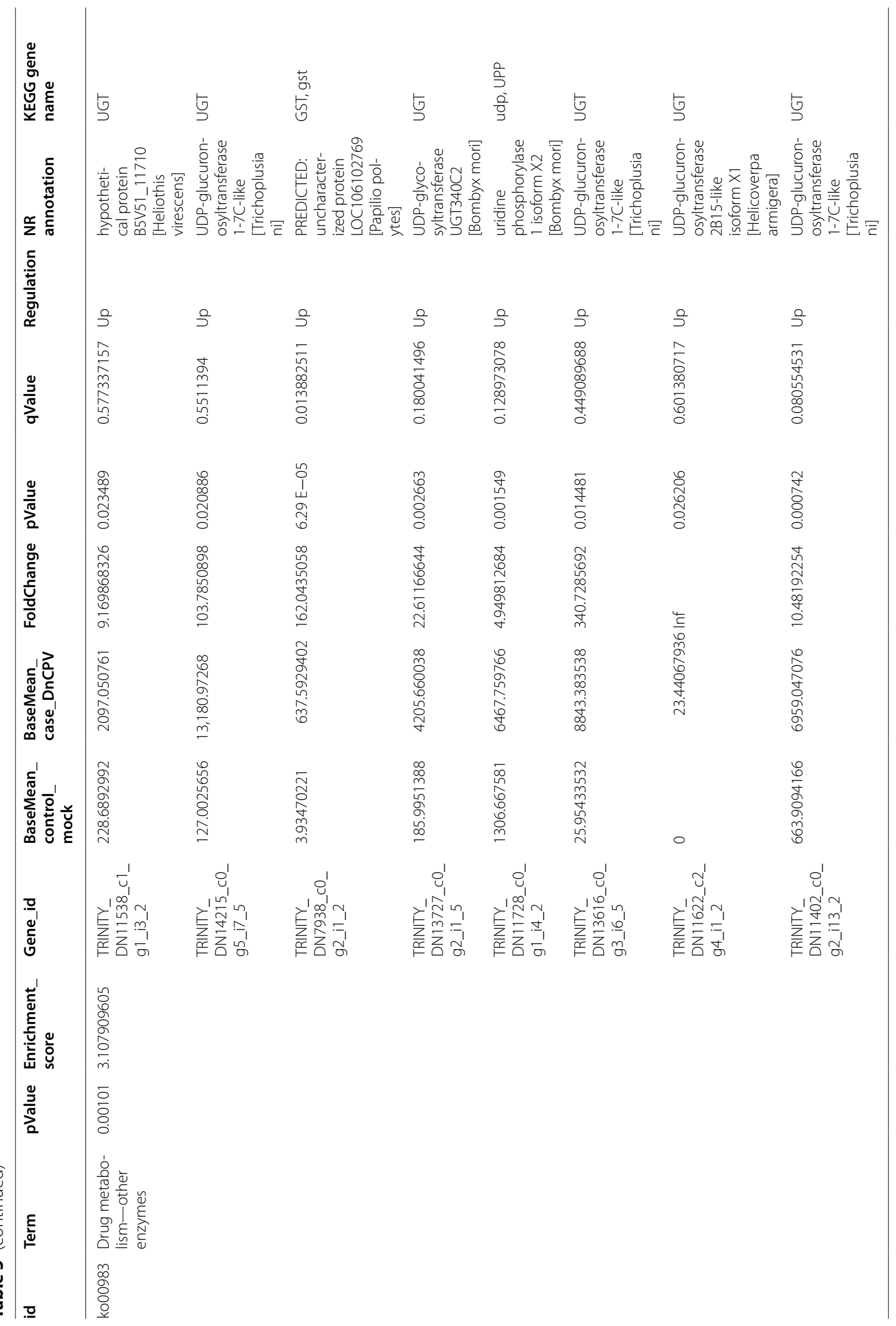


[44]. However, the interactions between UGT and cypovirus still remain unclear. In Table 5, there were the pathways and genes mentioned above and genes expression of each sample is shown in Additional file 1.

\section{Conclusion}

This study revealed substantial differences in the transcriptions of the $D$. nerii genes related to digestion, immunity, glycerophospholipid metabolism and toxic substances metabolism induced by DnCPV-23 replication. Findings obtained in this research further enriched the understanding of cypovirus-Spodoptera insect interactions in midgut and provided additional basic information for the future exploitation of DnCPV-23.

\section{Abbreviations}

DnCPV-23: Daphnis nerii Cypovirus-23; D. nerii: Daphnis nerii; PGRP: Peptidoglycan Recognition Protein;" CASP-8: Caspase-8.

\section{Supplementary Information}

The online version contains supplementary material available at https://doi. org/10.1186/s12985-021-01721-x.

Additional file 1. All the different expression genes in the midgut after DnCPV-23 infection.

\section{Acknowledgements}

Thanks to the molecular experiment platform provided by the Institute of Microbiology, Jiangxi Academy of Sciences.

\begin{abstract}
Authors' contributions
KW, YC designed and performed the experiments and analysed the data. ZZ was responsible for revising the manuscript. ZZ, GL and CJ collected Daphnis nerii larval. WJ and $\amalg$ provided suggestions. KW, YC and JL wrote the manuscript. JL, ZX, and MG supervised the project and revised the manuscript. All Authors have read and approved the final version of the manuscript.
\end{abstract}

\section{Funding}

This research was supported by the Doctoral Research Startup Project of Jiangxi Academy of Sciences (2019-XTPH1-04), the Doctoral Research Startup Fund of Jiangxi University of Traditional Chinese Medicine (2020BSZR012), the Natural Science Foundation of Jiangxi Province (20192ACB20008), the key Research and Development Project of Jiangxi Province (20192BBF60056) and the key Research and Development Project of Jiangxi Province (20202BBFL63050).

\section{Availability of data and materials}

The original data of the transcriptome will be released on 2021-10-05 or upon publicationhas, BioProject accession: PRJNA766516.

\section{Declarations}

Ethics approval and consent to participate Not applicable.

\section{Consent to publication}

Not applicable.

\section{Competing interests}

The authors declare that they have no competing interests.

\section{Author details}

${ }^{1}$ Institute of Microbiology, Jiangxi Academy of Sciences, No. 7777 Changdong Road, Nanchang 330096, China. ${ }^{2}$ State Key Laboratory of Virology, Wuhan Institute of Virology, Chinese Academy of Sciences (CAS), Wuhan 430071, China. ${ }^{3}$ School of Life Sciences, Jiangxi University of Traditional Chinese Medicine, Nanchang 330004, China.

Received: 13 August 2021 Accepted: 1 December 2021

Published online: 14 December 2021

\section{References}

1. Zhan Z, Guan L, Wang J, Liu Z, Guo Y, Xiao Y, Wang H, Jin L. Isolation and genomic characterization of a cypovirus from the oleander hawk moth, Daphnis nerii. J Invertebr Pathol. 2019;163:43.

2. Lei YL, Lin ZG. Bionomics of the oleander hawkmoth, Daphnis nerii. Chin J Appl Entomol. 2010;47:918-22.

3. Sun Y, Chen C, Gao J, Abbas MN, Kausar S, Qian C, Wang L, Wei G, Zhu BJ, Liu CL. Comparative mitochondrial genome analysis of Daphnis nerii and other lepidopteran insects reveals conserved mitochondrial genome organization and phylogenetic relationships. PLoS ONE. 2017;12:e0178773.

4. Lu Q, Ren F, Yan J, Zhang Y, Awais M, He J, Sun J. Alkaline phosphatase can promote the replication of Bombyx mori cypovirus 1 by interaction with its turret protein. Virus Res. 2021;292:198261.

5. Green TB, White S, Rao S, Mertens PP, Adler PH, Becnel JJ. Biological and molecular studies of a cypovirus from the black fly Simulium ubiquitum (Diptera: Simuliidae). J Invertebr Pathol. 2007;95:26-32.

6. Zhou Y, Qin T, Xiao Y, Qin F, Lei C, Sun X. Genomic and biological characterization of a new cypovirus isolated from Dendrolimus punctatus. PLoS ONE. 2014;9:1.

7. Zhang GB, Yang J, Qin FJ, Xu CR, Wang J, Lei CF, Hu J, Sun XL. A reverse genetics system for cypovirus based on a bacmid expressing T7 RNA polymerase. Viruses Basel. 2019;11.

8. Kuang WD, Zhan ZG, Guan LM, Wang JC, Yan CH, Chen JH, Li JH, Zhou X, Jin L. Study on proliferation characteristics of Daphnis nerii cypovirus-23 in Sf9 cells. J. Agric. Biotechnol. 2021;29:772-779 (in Chinese).

9. Jiang L, Li GX, Li CY, Robert RG, Gary WB. Growth characteristics and expression of recombinant proteins in three new cell lines from Manduca sexta (Lepidoptera:Sphingidae). Acta Entomol Sin. 2010;53:1227-32 (in Chinese).

10. Kolliopoulou A, Van Nieuwerburgh F, Stravopodis DJ, Deforce D, Swevers L, Smagghe G. Transcriptome analysis of Bombyx mori larval midgut during persistent and pathogenic cytoplasmic polyhedrosis virus infection. PLOS ONE. 2015:10:e0121447.

11. Guo R, Wang S, Xue R, Cao G, Hu X, Huang M, Zhang Y, Lu Y, Zhu L, Chen $F$, et al. The gene expression profile of resistant and susceptible Bombyx mori strains reveals cypovirus-associated variations in host gene transcript levels. Appl Microbiol Biotechnol. 2015;99:5175-87.

12. Gao K, Deng XY, Qian HY, Qin GX, Hou CX, Guo XJ. Cytoplasmic polyhedrosis virus-induced differential gene expression in two silkworm strains of different susceptibility. Gene. 2014;539:230-7.

13. Wu P, Wang X, Qin GX, Liu T, Jiang YF, Li MW, Guo XJ. Microarray analysis of the gene expression profile in the midgut of silkworm infected with cytoplasmic polyhedrosis virus. Mol Biol Rep. 2011;38:333-41.

14. Gao K, Deng XY, Qian HY, Qin G, Guo XJ. Digital gene expression analysis in the midgut of 4008 silkworm strain infected with cytoplasmic polyhedrosis virus. J Invertebr Pathol. 2014;115:8-13.

15. Jiang L, Peng ZW, Guo YB, Cheng TC, Guo HZ, Sun Q, Huang CL, Zhao P, Xia QY. Transcriptome analysis of interactions between silkworm and cytoplasmic polyhedrosis virus. Sci Rep. 2016; 6.

16. Wu P, Han S, Chen T, Qin G, Li L, Guo X. Involvement of microRNAs in infection of silkworm with bombyx mori cytoplasmic polyhedrosis virus (BmCPV). PLoS ONE. 2013; 8:e68209.

17. Wu P, Qin G, Qian H, Chen T, Guo X. Roles of miR-278-3p in IBP2 regulation and Bombyx mori cytoplasmic polyhedrosis virus replication. Gene. 2016:575:264-9.

18. Pan ZH, Wu P, Gao K, Hou CX, Qin GX, Geng T, Guo XJ. Identification and characterization of two putative microRNAs encoded by Bombyx mori cypovirus. Virus Res. 2017;233:86-94. 
19. Zhang Y, Cao G, Zhu L, Chen F, Zar MS, Wang S, Hu X, Wei Y, Xue R, Gong $C$. Integrin beta and receptor for activated protein kinase $C$ are involved in the cell entry of Bombyx mori cypovirus. Appl Microbiol Biotechnol. 2017;101:3703-16.

20. Wu P, Jie W, Shang Q, Annan E, Jiang X, Hou C, Chen T, Guo X. DNA methylation in silkworm genome may provide insights into epigenetic regulation of response to Bombyx mori cypovirus infection. Sci Rep. 2017;7:16013.

21. Zhang X, Zhang YS, Shi X, Dai K, Liang Z, Zhu M, Zhang ZY, Shen ZE, Pan J, Wang $C L$, et al. Characterization of the lipidomic profile of $B m N$ cells in response to Bombyx mori cytoplasmic polyhedrosis virus infection. Dev Comp Immunol. 2021;114.

22. Shrestha A, Bao K, Chen W, Wang P, Fei Z, Blissard GW. Transcriptional responses of the Trichoplusia ni Midgut to oral infection by the Baculovirus Autographa californica multiple Nucleopolyhedrovirus. J Virol. 2019;93.

23. Langmead B, Salzberg SL. Fast gapped-read alignment with Bowtie 2. Nat Methods. 2012;9:357-9.

24. Roberts $A$, Pachter $L$. Streaming fragment assignment for real-time analysis of sequencing experiments. Nat Methods. 2013;10:71-3.

25. Trapnell C, Williams BA, Pertea G, Mortazavi A, Kwan G, van Baren MJ, Salzberg SL, Wold BJ, Pachter L. Transcript assembly and quantification by RNA-Seq reveals unannotated transcripts and isoform switching during cell differentiation. Nat Biotechnol. 2010;28:511-5.

26. Anders $S$, Huber W. Differential expression analysis for sequence count data. Genome Biol. 2010;11.

27. Livak KJ, Schmittgen TD. Analysis of relative gene expression data using real-time quantitative PCR and the $2(T)(-$ Delta Delta $C)$ method. Methods. 2001;25:402-8

28. Rubinstein R. Characterization of the proteins and serological relationships of cytoplasmic polyhedrosis virus of Heliothis armigera. J Inverterb Pathol. 1983;42:292-4.

29. Gao K, Deng XY, Qian HY, Qin GX, Hou CX, Guo XJ. Cloning and expression analysis of a peptidoglycan recognition protein in silkworm related to virus infection. Gene. 2014;552:24-31.

30. Kurz CL, Charroux B, Chaduli D, Viallat-Lieutaud A, Royet J. Peptidoglycan sensing by octopaminergic neurons modulates Drosophila oviposition. Elife. 2017;6.

31. Neyen C, Runchel C, Schupfer F, Meier P, Lemaitre B. The regulatory isoform rPGRP-LC induces immune resolution via endosomal degradation of receptors. Nat Immunol. 2016;17:1150-8.

32. Jiang L, Liu W, Guo H, Dang Y, Cheng T, Yang W, Sun Q, Wang B, Wang Y, Xie E, Xia Q. Distinct functions of Bombyx mori peptidoglycan recognition protein 2 in immune responses to bacteria and viruses. Front Immunol. 2019;10:776.

33. Zhao $P$, Xia F, Jiang $L$, Guo H, Xu G, Sun Q, Wang B, Wang Y, Lu Z, Xia Q. Enhanced antiviral immunity against Bombyx mori cytoplasmic polyhedrosis virus via overexpression of peptidoglycan recognition protein S2 in transgenic silkworms. Dev Comp Immunol. 2018;87:84-9.

34. Cooper DM, Granville DJ, Lowenberger C. The insect caspases. Apoptosis. 2009;14:247-56.

35. Ma X, Li X, Dong S, Xia Q, Wang F. A Fas associated factor negatively regulates anti-bacterial immunity by promoting Relish degradation in Bombyx mori. Insect Biochem Mol Biol. 2015;63:144-51.

36. Stoven S, Silverman N, Junell A, Hedengren-Olcott M, Erturk D, Engstrom Y, Maniatis T, Hultmark D. Caspase-mediated processing of the Drosophila NF-kappaB factor Relish. Proc Natl Acad Sci U S A. 2003;100:5991-6.

37. Manniello MD, Moretta A, Salvia R, Scieuzo C, Lucchetti D, Vogel H, Sgambato A, Falabella P. Insect antimicrobial peptides: potential weapons to counteract the antibiotic resistance. Cell Mol Life Sci. 2021;78:4259-82.

38. Hua X, Li B, Song L, Hu C, Li X, Wang D, Xiong Y, Zhao P, He H, Xia Q, Wang F. Stimulator of interferon genes (STING) provides insect antiviral immunity by promoting Dredd caspase-mediated NF-kappaB activation. J Biol Chem. 2018:293:11878-90.

39. Zhu LY, Hu XL, Kumar D, Chen F, Feng YJJ, Zhu M, Liang Z, Huang LX, Yu L, $\mathrm{Xu}$ J, et al. Both ganglioside GM2 and cholesterol in the cell membrane are essential for Bombyx mori cypovirus cell entry. Dev Comp Immunol. 2018;88:161-8.

40. Nanbo A, Maruyama J, Imai M, Ujie M, Fujioka Y, Nishide S, Takada A, Ohba Y, Kawaoka Y. Ebola virus requires a host scramblase for externalization of phosphatidylserine on the surface of viral particles. PLoS Pathog. 2018;14:e1006848.

41. Basu NK, Kovarova M, Garza A, Kubota S, Saha T, Mitra PS, Banerjee R, Rivera J, Owens IS. Phosphorylation of a UDP-glucuronosyltransferase regulates substrate specificity. Proc Natl Acad Sci U S A. 2005;102:6285-90.

42. Bock KW. The UDP-glycosyltransferase (UGT) superfamily expressed in humans, insects and plants: animal-plant arms-race and co-evolution. Biochem Pharmacol. 2016;99:11-7.

43. Mackenzie PI, Hu DG, Gardner-Stephen DA. The regulation of UDPglucuronosyltransferase genes by tissue-specific and ligand-activated transcription factors. Drug Metab Rev. 2010;42:99-109.

44. Rowland A, Miners JO, Mackenzie PI. The UDP-glucuronosyltransferases: their role in drug metabolism and detoxification. Int J Biochem Cell Biol. 2013;45:1121-32.

\section{Publisher's Note}

Springer Nature remains neutral with regard to jurisdictional claims in published maps and institutional affiliations.

Ready to submit your research? Choose BMC and benefit from:

- fast, convenient online submission

- thorough peer review by experienced researchers in your field

- rapid publication on acceptance

- support for research data, including large and complex data types

- gold Open Access which fosters wider collaboration and increased citations

- maximum visibility for your research: over $100 \mathrm{M}$ website views per year

At BMC, research is always in progress.

Learn more biomedcentral.com/submissions 